\title{
Coherent Microscopy and Optical Coherence Tomography for Biomedical Applications
}

\author{
Jeremy M. Coupland \\ Tel.: ++44 (0)1509 227506; Fax: ++44 (0)1509 227502; Email: j.m.coupland@lboro.ac.uk \\ Wolfson School of Mechanical and Manufacturing Engineering, Loughborough University, UK
}

\begin{abstract}
In recent years many new methods of 3D optical imaging have been introduced that are applicable to the study of micro- and nano-scale flows. Coherent microscopy and optical coherence tomography join more established methods such as coherence scanning interferometry and confocal microscopy. These methods are very closely related and, using linear systems theory, can be compared in terms of their point spread and transfer functions. This paper introduces linear theory, demonstrates the main differences between the methods and discusses their use in micro- and nano-scale flow measurement. It is shown that coherent microscopy is currently the only method capable of single shot recording and consequently simultaneous whole-field flow measurement. Its use is limited to sparsely seeded flows however, such that individual particles can be identified. The other techniques provide increased 3D discrimination. Using a large numerical aperture, confocal microscopy and coherence scanning interferometry provide the most detailed 3D images making use the additional information available when the object is illuminated with plane waves propagating at different angles. In contrast optical coherence tomography uses the information that is available when the object is illuminated with different wavelengths. It is shown that the fundamental difference between these approaches is that the lateral and axial resolutions are decoupled in OCT making the technique easily scalable. This and the development of modern tunable laser sources, make OCT the method of choice for many biomedical applications.
\end{abstract}

Keywords: Holography, Digital Holography, Digital Holographic Microscopy, Optical Diffraction Tomography, Optical Coherence Tomography, Coherence Scanning Interferometry, Scanning White Light Interferometry, Confocal Microscopy, Impulse Response, Transfer function, Fourier Optics.

\section{Introduction}

Driven mainly by biomedical applications the optical microscope has been in constant development for over a century. Significant milestones include the imaging theory of Abbe, the phase contrast technique introduced by Zernike, and the confocal microscope, proposed by Minsky (Minsky 1988), which has the capability to produce 3D sectioned images. More recently we have witnessed the development of coherent microscopy and other techniques that have similar 3D capability. In particular, Optical Coherence Tomography (OCT) has produced truly remarkable 3D images of the retina and Doppler OCT has been used to estimate blood flow within tissue (Fercher et. al. 2003).

Although these microscopy techniques might seem quite disparate both in configuration and application, they are similar in many important respects. With the exception of fluorescent confocal microscopy, elastically scattered light is collected from the object of interest and interferometric methods are used to record the phase and the amplitude of the scattered field. In an abstract sense, all optical techniques derive information from the response of the object to a set of optical stimuli with known spatial and temporal characteristics. Hence, it is only the scanning methods that provide the illuminating fields and those used to record the corresponding responses, which differentiate the various techniques.

In previous papers (Coupland and Lobera 2008, Ruiz et. al. 2010) we have applied scalar diffraction theory to many optical imaging 
techniques, characterizing them as 3D linear systems. In addition we have also considered image enhancing methods such as phase contrast etc. as 3D linear filtering operations. (Lobera and Coupland 2008). Although the details of this analysis are beyond the scope of this paper, the main results provide a useful insight into the capability of optical techniques and their use as means to study micro- and nano-scale flows.

Before beginning the discussion it is important to state that linear theory rests on the assumption of weak scattering (the Born approximation, - see Wolf 1969). In effect it is assumed that the scattered field is a small perturbation to the field that illuminates the object as is often the case in flow measurements where the fluid is usually sparsely seeded with small tracer particles. Although the Born approximation is not strictly justified in dense tissue, linear theory provides a reasonable estimate of imaging performance as multiple scattering typically raises noise levels.

According to linear systems theory the process of imaging can be thought of as a filtering operation that is characterised in the space domain as the 3D convolution integral,

$$
\mathrm{I}(\mathbf{r})=\int_{-\infty}^{+\infty} \mathrm{H}\left(\mathbf{r}-\mathbf{r}^{\prime}\right) \Delta\left(\mathbf{r}^{\prime}\right) \mathrm{d}^{3} \mathbf{r}^{\prime}
$$

where $\mathrm{H}(\mathbf{r})$ is the point spread function (PSF) and $d^{3} r^{\prime}$ represents the scalar $d r_{x}{ }^{\prime} d_{y}{ }^{\prime} d^{2} r_{z}$. The function $\Delta(\mathbf{r})$, represents the object and in this paper will be defined as the refractive index contrast,

$$
\Delta(\mathbf{r})=1-\mathrm{n}^{2}(\mathbf{r})
$$

where, $\mathrm{n}(\mathbf{r})$ is the refractive index.

The PSF can be thought of as the image of an idealised point scattering particle and its dimensions effectively define the resolution of the system. Alternatively, in the spatial frequency domain the convolution can be written more straightforwardly as the product,

$$
\tilde{\mathrm{I}}(\mathbf{k})=\tilde{\mathrm{H}}(\mathbf{k}) \tilde{\Delta}(\mathbf{k})
$$

where tilde represents Fourier transformation and $\tilde{\mathrm{H}}(\mathbf{k})$ is the transfer function of the system. The transfer function describes how the phase and amplitude of the individual spatial frequency components that make up the object function are changed by the instrument/reconstruction process.

It is more intuitive to characterise the response of optical systems in the spatial frequency domain (k-space) and then go on to calculate the corresponding PSF. This is because each spatial frequency component of the object (represented by a point in k-space) can be considered as a Bragg grating. If a Bragg grating is illuminated by a monochromatic plane wave, it will only reflect at a single a single angle of incidence. If at this orientation the illuminating wave and the reflected wave are represented by the wave vectors $\mathbf{k}_{\mathrm{i}}$, and $\mathbf{k}_{\mathrm{r}}$, then they are related to the grating vector $\mathbf{k}_{\mathrm{g}}$ such that,

$$
\mathbf{k}_{\mathrm{r}}-\mathbf{k}_{\mathrm{i}}=\mathbf{k}_{\mathrm{g}}
$$

Under the assumption of weak scattering it can be shown that the amplitude of the reflected wave is directly proportional to the refractive index contrast. Consequently it is possible to measure the amplitude of a particular spatial frequency component by measuring the response of the object to appropriately chosen illumination and observation directions. It is not generally possible however, to measure all spatial frequency components due to limitations in the wavelength of available sources and restrictions in the possible illumination and observation directions. Even when viewing restrictions are removed, it is straightforward to show that the quantity of data is overwhelming for volumes greater than about $1 \mathrm{~mm}^{3}$.

In the following, the main methods used in 3D imaging systems are compared in terms of PSF and transfer characteristics and later their potential as measurement tools for the study of micro- and nano-flow studies will be discussed. 


\section{Coherent Microscopy}

Coherent microscopy, or digital holographic microscopy as it is now frequently called, is shown schematically in figure 1 . In this arrangement the laser is focused in the back focal plane of the objective such that the object is illuminated by a plane wave.

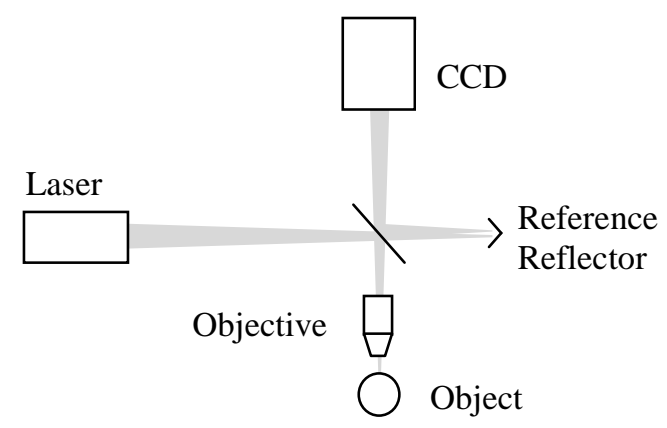

Fig. 1. Coherent microscopy configuration

The light scattered from the object passes back through the objective and forms an image on the CCD. The image is mixed with a coherent reference wave of appropriate divergence and tilt such that plane carrier fringes are observed in the intensity recorded by the CCD. Using 2D Fourier transformation it is straightforward to decompose the image into its constituent wave vector components.

In coherent microscopy we have a single wavelength and the aperture of the objective lens only collects the plane wave components of the scattered field over a cone defined by the numerical aperture (NA). The scattered wavevectors $\mathbf{k}_{\mathrm{r}}$ that are accepted by the system are represented by a corresponding part of an infinitely thin spherical shell with a radius equal to the wavenumber $\mathrm{k}_{0}=1 / \lambda$. The corresponding transfer function is shown schematically in figure 2 .

Mathematically, the transfer function can be expressed as the function,

$$
\tilde{\mathrm{H}}(\mathbf{k})=\delta\left(|\mathbf{k}|-\mathrm{k}_{0}\right) \operatorname{step}\left(\mathbf{k} . \hat{\mathbf{o}}-\mathrm{k}_{0} \sqrt{1-\mathrm{NA}^{2}}\right)
$$

where $\hat{\mathbf{o}}$ is a unit vector in the direction of the viewing axis and where $\delta(\mathbf{x})$ and $\operatorname{step}(\mathrm{x})$ are the Dirac delta function and Heaviside step function respectively.

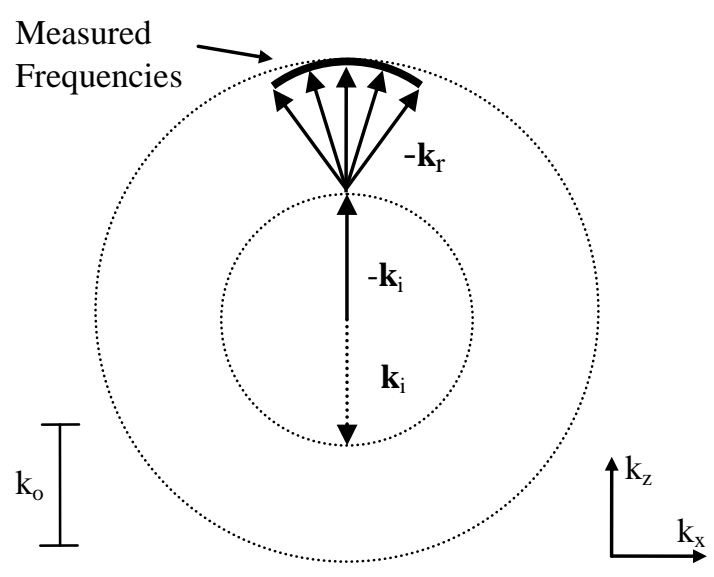

Fig. 2. Transfer function of a coherent microscope

Inverse Fourier transformation of this expression gives the PSF. This has been computed for a coherent microscope with $\mathrm{NA}=0.55$ operating at $\lambda=600 \mathrm{~nm}$ and a section through this distribution is shown in figure 3 .

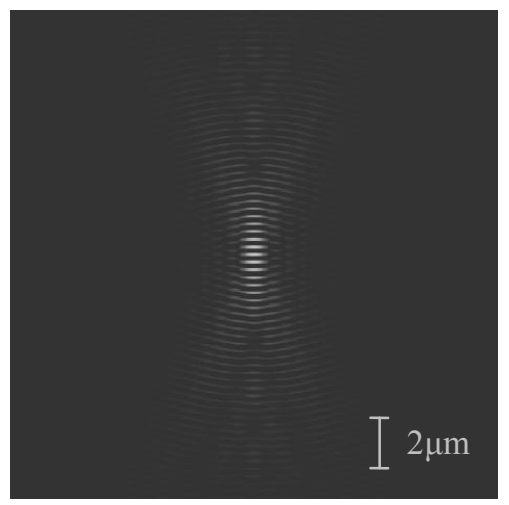

Fig. 3. PSF of a coherent microscope

It is noted that the PSF is appears similar to a focused beam and as such the intensity of the of the PSF decreases as the square of the distance in the observation direction from its centre. This means that the total intensity in any plane perpendicular to the optical axis remains constant and for this reason it has been stated that coherent microscopy does not contain 3D information (Fercher, 2003). Nevertheless, if it is used to investigate sparsely seeded flows individual particles can 
distinguished as bright points in the reconstruction and their $3 \mathrm{D}$ position can be measured (Wormald and Coupland, 2009).

\section{Optical Coherence Tomography}

There are many implementations of optical coherence tomography (OCT), but in essence they all measure the coherent response of an object when the wavelength of the illumination is varied. An OCT system can be considered as the superposition of images from a coherent microscope made at different wavelengths as shown schematically for illumination with 4 different wavenumbers in figure 4 . In a real system the illumination wavelength is scanned and providing the frame rate of the CCD is sufficient, the measured frequencies form a continuum. In this case the transfer function of the system can be written as the superposition integral,

$$
\tilde{\mathrm{H}}(\mathbf{k})=\int_{-\infty}^{+\infty} \mathrm{S}\left(\mathrm{k}_{0}\right) \delta\left(|\mathbf{k}|-\mathrm{k}_{0}\right) \operatorname{step}\left(\mathbf{k} . \hat{\mathbf{o}}-\mathrm{k}_{0} \sqrt{1-\mathrm{NA}^{2}}\right) \mathrm{dk}_{0}
$$

where $S\left(k_{0}\right)$ is the amplitude spectrum of the source expressed as a function of wavenumber.

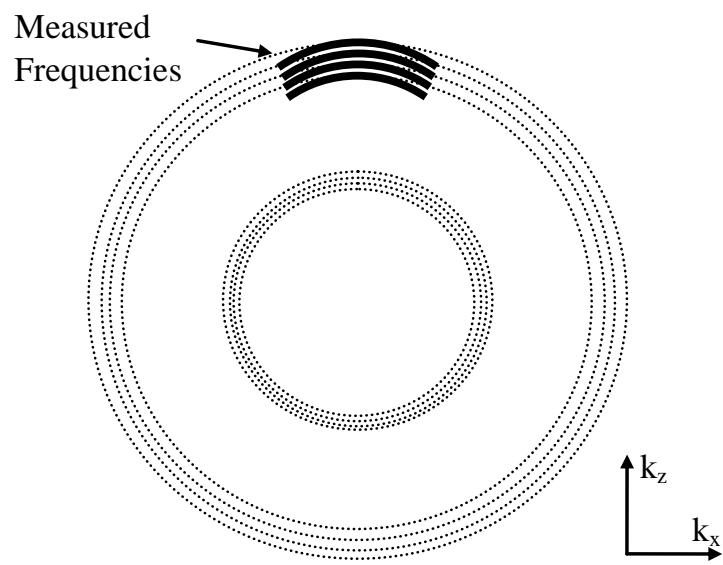

Fig. 4. Transfer function of an OCT system

Inverse Fourier transformation of this expression gives the PSF that is shown in figure 5. For the sake of comparison, the PSF has been computed for a system with the previous $\mathrm{NA}=0.55$ operating with the same mean wavelength of $\lambda=600 \mathrm{~nm}$ and a Gaussian spectral distribution with bandwidth of $100 \mathrm{~nm}$ (FWHH@1/e ${ }^{2}$.

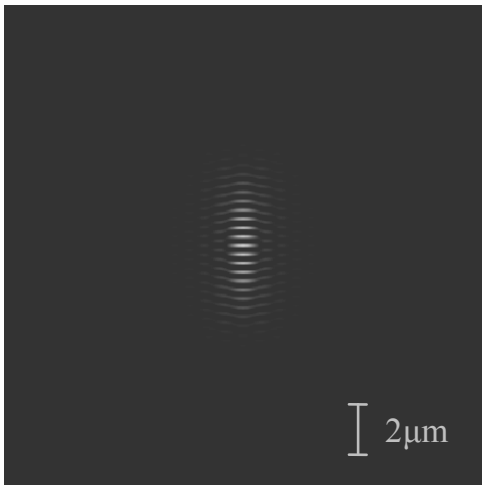

Fig. 5. PSF of an OCT system

It can be seen that in contrast to figure 3 the total intensity of the PSF reduces in planes orthogonal to the viewing direction and as such OCT provides greatly enhanced 3D information and is therefore a truly tomographic technique. In OCT the extent of the PSF in the axial (i.e. observation) and lateral (perpendicular to the observation) directions are inversely proportional to the source bandwidth and the NA respectively. It is important to point out that most commercial OCT systems operate with much reduced aperture $(\mathrm{NA}=0.01)$ resulting in a PSF with proportionately larger lateral dimensions.

\section{Coherence Scanning Interferometry and Confocal Microscopy}

Coherence Scanning Interferometry (CSI) and confocal microscopy can also be considered to be tomographic techniques and are discussed together in this section because they both provide additional 3D information that is beyond that obtainable using coherent microscopy and OCT. This information is the response of the object to illuminating plane waves at propagating at different angles. Interestingly, both CSI and confocal microscopy pre-date coherent microscopy and OCT.

In CSI the object is illuminated by a wideband distributed source (halogen or LED) through a Mirau interference objective as 
shown in figure 6 . Interference between the light reflected from a reference surface (within the Mirau objective) and that from the objective is recorded in the by the CCD as the object is scanned through focus.

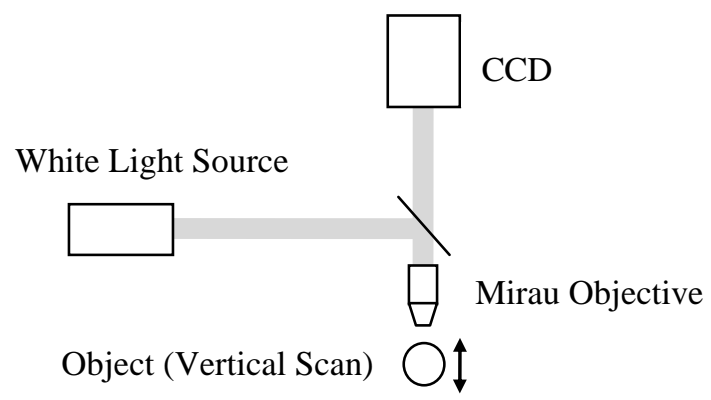

Fig. 6. CSI configuration

Considering first the distributed nature of the source, the interference fringes generated by a quasi monochromatic CSI can be considered to be due to the superposition of coherent recordings made different plane wave propagating at different angles. The response is illustrated schematically for 5 different plane wave illuminations in figure 7 .

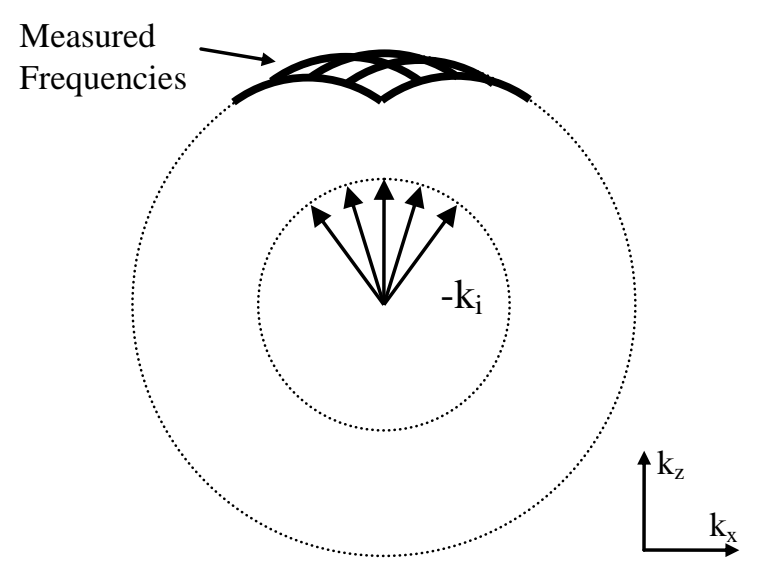

Fig. 7. Transfer function of quasimonochromatic CSI system

In practice if a distributed source and appropriate condenser lenses are used to fill the aperture of the objective, the transfer function becomes a continuum within the region shown.

If we now consider a polychromatic source the superposition results in the transfer function shown schematically in Figure 8.

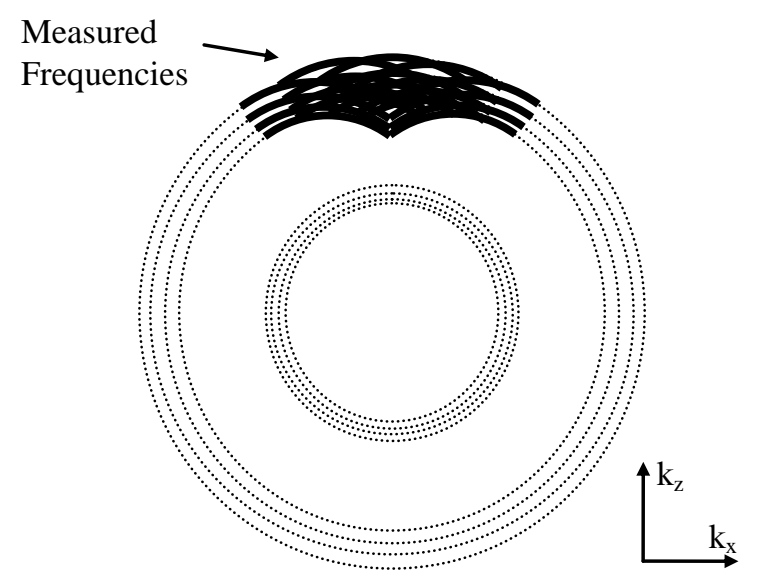

Fig. 8. Transfer function of a polychromatic CSI system

In practice a source will have a smooth spectrum and the transfer function will be a continuum defined by,

$$
\tilde{\mathrm{H}}(\mathbf{k})=\int_{-\infty}^{+\infty} \mathrm{S}\left(\mathrm{k}_{0}\right) \mathrm{A}(\mathbf{k}) \mathrm{dk}_{0}
$$

where the function $\mathrm{A}(\mathbf{k})$ is defined by the convolution,

$$
\begin{aligned}
& \mathrm{A}(\mathbf{k})=\int_{-\infty}^{+\infty} \delta\left(\left|\mathbf{k}^{\prime}\right|-\mathrm{k}_{0}\right) \operatorname{step}\left(\mathbf{k}^{\prime} \cdot \hat{\mathbf{o}}-\mathrm{k}_{0} \sqrt{1-\mathrm{NA}^{2}}\right) \\
& \times \delta\left(\left|\mathbf{k}-\mathbf{k}^{\prime}\right|-\mathrm{k}_{0}\right) \operatorname{step}\left(\left(\mathbf{k}-\mathbf{k}^{\prime}\right) \cdot \hat{\mathbf{o}}-\mathrm{k}_{0} \sqrt{1-\mathrm{NA}^{2}}\right) \mathrm{d}^{3} \mathbf{k}^{\prime}
\end{aligned}
$$

For comparison, the PSF has been computed in figure 9 for a CSI with $\mathrm{NA}=0.55$ operating with the same mean wavelength of $\lambda=600 \mathrm{~nm}$ and a Gaussian spectral distribution with bandwidth of 100nm (FWHH@1/ ${ }^{2}$ ) .

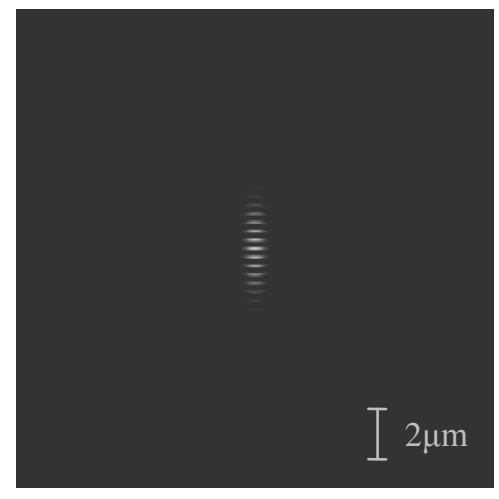

Fig. 9. PSF of a CSI system 
It can be seen that the lateral dimensions of the PSF are approximately half that of the coherent microscope and the OCT system while the axial extent is slightly less.

Interestingly, the PSF of a laser confocal microscope (as shown in figure 10) is directly related to that of CSI (Coupland and Lobera, 2008).

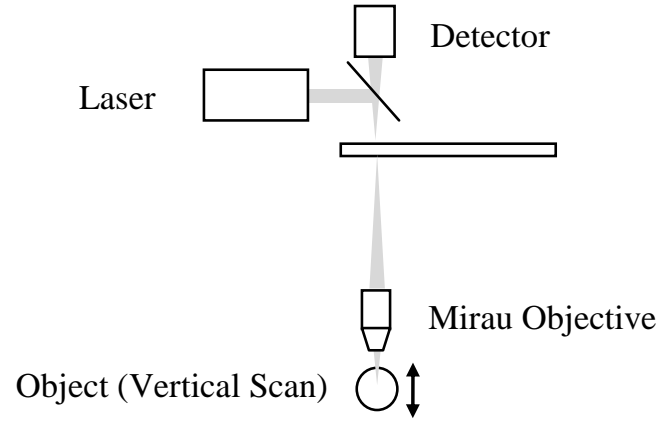

Fig. 10. Confocal microscope

In confocal microscopy however, the detection process is not usually coherent (i.e. not interferometric) and consequently the PSF takes the form of the envelope of the PSF of a quassi-monochromatic CSI of the same numerical aperture. For a given NA both CSI and confocal microscopy will resolve a horizontal feature of twice the spatial frequency of that which can be observed using an OCT or coherent microscope. It is noted that at low NA the axial extent of the PSF for both CSI and OCT is inversely proportional to the source bandwidth. For quassimonochromatic CSI and confocal microscopy however, the axial extent is inversely proportional to the squared NA. For broadband CSI systems the axial extent is either band width or NA limited and for typical systems a crossover is observed when $\mathrm{NA} \approx 0.3$.

\section{Flow Measurement}

It can be seen from the discussions above that it is possible to compare 3D optical imaging techniques using linear systems theory. In essence, the PSF shows the appearance of a small point-like particle and has been calculated for each technique. This is of course of great concern when deriving flow velocity measurements from seeded fluids.

It is noted that only optical configurations that operate in backscatter have been considered. In a backscatter geometry the PSF has a phase variation that has a spatial frequency that is slightly less than half the mean wavelength along its central axis. As a particle moves its reconstructed image will move in sympathy and its velocity can be deduced by estimating the displacement of its image using correlation for example. This is the basis of holographic particle image velocimetry (Coupland and Halliwell, 1992). Because of the phase variation within the PSF however, the fluid velocity at a point in space can also be found by measuring the change in phase at that point. This is the basis of laser Doppler velocimetry (Durst et. al. 1973) and Doppler OCT (Fercher, 2003).

Returning to the form of the PSF, there is a clear distinction between coherent microscopy and the other techniques, OCT, CSI and confocal microscopy. In the PSF of coherent microscopy, the total intensity in any plane perpendicular to the central axis is equal. Consequently individual images of particles can only be distinguished if the particles are relatively sparse. Analysis shows that an upper limit on the total number of particles that can be identified in this way is proportional to the number of pixels required to record an optimally sampled holographic image (Coupland et. al., 2000). In practice however, additional noise sources reduce this limit and typically around $10^{4}$ particles $/ \mathrm{mm}^{3}$ can be identified.

The PSFs of OCT, CSI and confocal microscopy reduce much more quickly in the axial direction than those of coherent microscopy and consequently many more particles can be distinguished per unit volume. The number is inversely proportional to the volume of the PSF. For the case of the CSI PSF shown in figure 9., we might expect around $10^{8}$ particles per $\mathrm{mm}^{3}$ while for a lower NA OCT system $(\mathrm{NA}=0.01)$ might return $10^{4}$ particles per $\mathrm{mm}^{3}$.

If the number concentration is increased slightly the images of particles will overlap 
and interfere. The reconstructed image will therefore take on a "speckled" appearance. With the tomographic techniques, OCT, CSI and confocal microscopy, it would be possible to identify local changes in number density as the speckle will become proportionately brighter or darker. This is not true, for the case of coherent microscopy. The decay of the PSF in the axial direction is much slower than the tomographic techniques and the 3D information is lost. For this reason it is necessary to use a tomographic method to image through tissue. As the number concentration or equivalently the scattering coefficient increases however, the assumption of weak scattering (Born approximation) breaks down and the effects of multiple scattering are observed. Typically this increases background noise in the reconstruction but can cause phantom images in some instances.

\section{Practical Issues}

The previous sections have compared the theoretical performance of the various approaches to 3D imaging. It is noted that the tomographic techniques provide superior performance to coherent microscopy but require addition images to be taken with different illumination conditions. This additional information comes at the expense of time and frequently it is necessary to compromise performance.

Although coherent microscopy can only be used to study relatively sparsely seeded flows, it is currently the only flow mapping technique capable of simultaneous whole-field flow measurement. Standard particle image velocimetry cameras and double pulsed laser sources make it possible to record pairs of holograms separated by $1-2 \mu$ s at megapixel resolution and at more than 1000 frame pairs per second. For micro-flow measurement this means a maximum flow velocity of approximately $1-10 \mathrm{~m} / \mathrm{s}$ depending on the configuration.

As discussed earlier, to image through scattering media or increase the data density by introducing more seeding particles it is necessary to use a tomographic technique.

Doppler OCT is now used routinely, both to image and measure blood flow within the retina. In its original form OCT used a configuration similar to low NA CSI and used a broadband incoherent source. This configuration is referred to as space domain OCT and required slow mechanical scanning. Commercial swept-source OCT systems now exploit fast scanning laser sources to measure the backscattered light scattering along the path of a weakly focused beam. 2D scanning mirrors are required to produce a complete $3 \mathrm{D}$ image. Typically the spectral scan rate is around $100 \mathrm{kHz}$ and this limits the maximum velocity to around $50 \mathrm{~mm} / \mathrm{s}$. Although, only the velocity component along the viewing direction is measured and this generally varies with position the $3 \mathrm{D}$ flow field can be synthesized from 3 or more data sets.

The methods of CSI and confocal microscopy have been included in this paper because of their close relationship to the other techniques. As described in section 3 both quasi-monochromatic CSI and confocal microscopy derive 3D information by illuminating the object with plane waves from different angles within the numerical aperture of the objective. Additionally, broadband CSI exploits spectral information like OCT. According to the linear theory, CSI and confocal microscopy will work with more densely seeded flows than coherent microscopy and offer significantly better resolution than OCT. Nevertheless OCT remains the method of choice for in-vivo flow measurement for several practical reasons. First, as mentioned previously, swept source OCT is clearly superior to the mechanical scanning of CSI. Second, in-vivo imaging generally requires propagation through a rough distorting surface (e.g. the epidermis) and this ruins the fidelity of the $3 \mathrm{D}$ reconstructions from both CSI and confocal microscopy at large NA. In addition, the object might exhibit significant dispersion (e.g. in the eye). In this case CSI can be used at small NA provided that the bandwidth is sufficiently small in which case it will perform identically to (but slower than) OCT. 


\section{Conclusion and Discussion}

This paper has compared the 3D imaging methods, coherent microscopy, OCT, CSI and confocal microscopy with reference to their point spread and transfer characteristics. There is a major distinction between coherent microscopy, that records a single coherent image at a single wavelength, and the other, tomographic, techniques that construct an image from a range of wavelengths and illumination conditions. There is also a major distinction between OCT and all the other methods. In coherent microscopy, CSI and confocal microscopy the methods the lateral resolution is proportional to the NA, but the depth resolution is proportional to the bandwidth of the source in OCT as opposed to the squared NA. This decoupling allows the PSF of OCT to be tailored to suit different scales. It is this flexibility, plus the efficiency of swept-source scanning that makes OCT such a powerful tool for biomedical applications.

\section{References}

Coupland, J.M. and N.A. Halliwell, 1992, Particle image velocimetry: three-dimensional fluid velocity measurements using holographic recording and optical correlation. Appl. Opt. 131(8), 1005-1007.

Coupland, J.M,, Lobera, J., and Halliwell N.A., 2000, Fundamental limitations to the spatial resolution and flow volume that can be mapped using holographic particle image velocimetry, Proc. of Europto/SPIE conference on Optical Diagnostics for Industrial Applications, Glasgow (UK), pp.90-102.

Coupland, J.M., Lobera, J., 2008, Holography, tomography and 3D microscopy as linear filtering operations. Meas. Sci. Technol. 19.

Durst, F., A. Melling, and J. Whitelaw. 1981. Principles and practice of laser Doppler anemometry. Academic Press.

Fercher, A.F., Drexler, W., Hitzenberger, C.K., Lasser, T., 2003, Optical coherence tomography- principles and applications, Reports on Progress in Physics, 66, 239-303.

Lobera, J., Coupland, J.M., 2008 Contrast enhancing techniques in digital holographic microscopy Meas. Sci. Technol. 19.

Minsky, M., 1988, Memoir On Inventing The Confocal Scanning Microscope, Scanning 10(4) 128-138.

Ruiz, P.D., Huntley, J.M., Coupland, J.M., 2010, Depth-resolved imaging and displacement measurement techniques viewed as linear filtering operations, Exp. Mech. 50(7).

Wolf E., 1969, Three-dimensional structure determination of semi-transparent objects from holographic data, Optics Communications 1(4), 153-156.

Wormald, S.A., Coupland, J.M., 2009, Particle image identification and correlation analysis in microscopic holographic particle image velocimetry, Appl. Opt., 48(33), 6400-6407. 